\title{
New diversity form of ice polymorphism: Discovery of second hydrogen ordered phase of ice $\mathrm{VI}$
}

\section{Ryo Yamane ( $\nabla$ r.yamane@issp.u-tokyo.ac.jp )}

The University of Tokyo

\section{Kazuki Komatsu}

The University of Tokyo https://orcid.org/0000-0003-3573-9174

\section{Jun Gouchi}

University of Tokyo

\section{Yoshiya Uwatoko}

The University of Tokyo https://orcid.org/0000-0002-1148-4632

\section{Shinichi Machida}

Neutron Science and Technology Center, CROSS

\section{Takanori Hattori}

Japan Proton Accelerator Research Complex (J-PARC) Center, Japan Atomic Energy Agency

\section{Hayate Ito}

The University of Tokyo

\section{Hiroyuki Kagi}

The University of Tokyo

\section{Article}

Keywords: ice polymorphism, hydrogen-ordered phases, polymorphic structures

Posted Date: August 28th, 2020

DOI: https://doi.org/10.21203/rs.3.rs-64673/v1

License: (c) (1) This work is licensed under a Creative Commons Attribution 4.0 International License.

Read Full License

Version of Record: A version of this preprint was published at Nature Communications on February 18th, 2021. See the published version at https://doi.org/10.1038/s41467-021-21351-9. 


\section{Abstract}

Ice exhibits extraordinary structural variety in its polymorphic structures. The existence of a new form of diversity in ice polymorphism has recently been debated in both experimental and theoretical studies, questioning whether hydrogen-disordered ice can transform into multiple hydrogen-ordered phases, contrary to the known one-to-one correspondence between disordered ice and its ordered phase. Here we report a new high-pressure phase, ice XIX, which is a second hydrogen-ordered phase of ice VI. This is the first discovery to demonstrate that disordered ice undergoes different manners of hydrogen ordering, which are thermodynamically controlled by pressure in the case of ice VI. Such multiplicity can appear in all disordered ice, and it widely provides a new research approach to deepen our knowledge, for example of the crucial issues of ice: the centrosymmetry of hydrogen-ordered configurations and potentially induced (anti-)ferroelectricity. Ultimately, this research opens up the possibility of completing the phase diagram of ice.

\section{Introduction}

To date, more than 20 crystalline and amorphous phases of ice have been reported ${ }^{1}$. This extraordinary polymorphism makes ice unique with its universality and has inspired many studies in the wide-ranging fields of material science and Earth and planetary science. The structural variety of ice arises from the geometric flexibility of hydrogen bonds and hydrogen order-disorder phase transition ${ }^{2}$, and hydrogen ordering in ice structure also induces drastic changes in the dynamic/static properties of ice along with the phase transition, such as immobilization of molecular rotation ${ }^{3}$ and ferro- or antiferroelectrically aligned molecular structures ${ }^{4-6}$. A prominent unsolved question ${ }^{7}$ concerning the structural diversity induced by hydrogen ordering is whether a hydrogen-disordered phase of ice transforms into only one hydrogen-ordered phase, as inferred from the currently known phase diagram of ice, although the energies of its possible hydrogen configurations are close because of the inherent geometric frustration of the ice lattice ${ }^{8-12}$. Recent experiments on a high-pressure hydrogen-disordered phase, ice VI, revealed an unknown hydrogen-ordered form $\left(\beta-\mathrm{XV}^{13}\right)$ besides the known ordered phase, ice $\mathrm{XV} .{ }^{14}$ Although the unknown ordered form would be a counterexample of the question, it has not been clarified whether $\beta$-XV is a distinct crystalline phase to be assigned as a new Roman numeral label due to lack of experimental evidence ${ }^{7,13,15-17}$. Herein we report a second hydrogen-ordered phase for ice VI, ice XIX, unambiguously demonstrated by in-situ dielectric and neutron diffraction measurements under high pressure. The phase boundary between ice $\mathrm{VI}$ and ice XIX shows that ice VI contracts upon hydrogen ordering, which thermodynamically stabilizes ice XIX in the higher-pressure region compared to ice XV because of its smaller volume than ice $X V^{8,14,18}$. The pressure-induced multiplicity of hydrogen-ordered phases, also theoretically suggested in other ice polymorphs ${ }^{11}$, can induce (or control) the different manners of hydrogen ordering of ice. Thus, this study demonstrates a hitherto undiscovered polymorphism of ice.

\section{Results And Discussion}


Comprehensive observation of hydrogen ordering in ice VI was conducted by dielectric experiments in the pressure range $0.88-2.2 \mathrm{GPa}$. Ice VI was initially obtained at room temperature and its dielectric properties were determined in both cooling and heating runs in the temperature range 100-150 K, using a newly developed pressure cell (see Methods). After the heating runs, the sample was subsequently heated to room temperature for annealing. Then, the sample was compressed again, and dielectric measurements were conducted at different pressures (Fig. 1).

Phase transitions from ice VI to its hydrogen-ordered phases were observed at around 120-130 K, along with sudden weakening of the dielectric response of ice VI with decreasing temperature (Fig. 2a and b). Hydrogen ordering of ice suppresses reorientation of water molecules which induces the dielectric response of ice ${ }^{2,19}$. We defined the disorder-order phase-transition temperatures from ice $\mathrm{VI}$ to its hydrogen-ordered phases as the starting temperature at which the slope $(\mathrm{d} / / \mathrm{d} T ;$ : . dielectric-loss peak intensity) changes (Fig. 2b). The slope of the obtained phase boundary, i.e. $\mathrm{d} T / \mathrm{d} P$, between ice $\mathrm{VI}$ and its hydrogen-ordered phases changes from negative to positive at around 1.6 GPa with increasing pressure (Fig. 1). Based on the Clausius-Clapeyron relationship, i.e. $\mathrm{d} T / \mathrm{d} P=\Delta V / \Delta S$, this sign change for $\mathrm{d} T / \mathrm{d} P$ strongly indicates that ice VI has two different hydrogen-ordered phases with opposite signs for $\Delta V$, because $\Delta S<0$ generally holds for hydrogen ordering. Since the currently known hydrogen ordering from ice $\mathrm{VI}$ to ice $\mathrm{XV}$ shows a positive volume change (observed at the lower pressure, $0.4 \mathrm{GPa}^{8}$ ), ice $\mathrm{XV}$ is in the lower pressure region and the hydrogen-ordered phase in the higher-pressure region is a new phase, ice XIX, which has a smaller volume than ice VI and also ice XV. The appearance of ice XIX is governed by the $P V$ term in the Gibbs energy expression, because the volume contraction thermodynamically stabilises ice XIX compared to ice XV. In this context, the phase boundary between ice XV and ice XIX should have a slope rather than lie horizontally as suggested previously ${ }^{13,16}$, because ice $X V$ has a larger volume than ice XIX (the supposed phase boundary in Fig. 1 is shown vertically to emphasise this point). It is noteworthy that the phase transition between ice VI and XIX showed hysteresis for the transition temperature (Supplementary Fig. 1). This first-order phase transition is consistent with the sudden change in dielectric properties between ice VI and ice XIX (Fig. 2).

Neutron diffraction experiments were conducted at 1.6 and $2.2 \mathrm{GPa}$ to confirm whether ice XIX is a hydrogen-ordered crystalline phase distinct from ice XV. Both cooling and heating runs were conducted at each pressure in the temperature range $80-150 \mathrm{~K}$.

A transition from ice VI to ice $\mathrm{XIX}$ was also observed in the neutron diffraction experiments, as appearance of new peaks due to symmetry lowering (Fig. 3a). Some of the new peaks, e.g. those at $2.20 \AA$ and $2.26 \AA$ (indicated by blue triangles in Fig. 3a), cannot be assigned to the unit cell of ice XV; instead, they can be assigned to an expanded $\sqrt{2} \times \sqrt{2} \times 1$ cell with respect to the unit cell of ice $\mathrm{VI}$ (the unit cell of ice $X V$ has a $1 \times 1 \times 1$ cell with respect to that of ice $\mathrm{VI}$ ). This is unambiguous evidence that the hydrogen-ordered phase found in the higher-pressure region is a crystalline phase distinct from ice $\mathrm{XV}$, and that ice VI has two different types of hydrogen ordering. The reflection conditions show that the unit cell of ice XIX has a primitive lattice. The reduced unit cell parameters of ice XIX, $a$ and $c$, corresponding Loading [MathJax]/jax/output/CommonHTML/fonts/TeX/fontdata.js pectively, upon hydrogen ordering (Fig. 3b); this 
tendency was also observed at $2.2 \mathrm{GPa}$. A comparison of the temperature dependences of $c / a$ at 1.6 and 2.2 GPa (Fig. 3c) showed that the phase-transition temperature at $2.2 \mathrm{GPa}$ was at about $7 \mathrm{~K}$ higher than that at $1.6 \mathrm{GPa}$. This result is consistent with the phase boundary between ice VI and XIX obtained by the dielectric experiments. On the other hand, no significant volume change was observed in our neutron diffraction experiments, in contrast to the expected negative volume change $(\Delta V<0)$ upon hydrogen ordering, probably due to the small volume contraction.

For the structure analysis of ice XIX, we considered candidates of its space group based on the groupsubgroup relationship between ice $\mathrm{VI}$ and $\mathrm{XIX}$, in addition to the experimentally confirmed reflection conditions. There are 36 subgroups for the space group of ice $\mathrm{Vl}, \mathrm{P}_{2} / \mathrm{nmc}$, considering the primitive unit cell of ice XIX. Among them, thirteen space groups, having $h 0 l: h+l=2 n$ and $0 k l: k+1=2 n$ reflection conditions, can be excluded from the observed reflection conditions. We conducted Rietveld analyses using structural models with 18 space groups of the remaining candidates, except for the lowersymmetry space groups: $P C, P 2_{1}, P 2, P \overline{1}$ and $P 1$-this cut-off is based on indices of the subgroups of $P 4_{2} / n m c$ (see details in Supplementary information). Notably, we do not rule out the possibility that the actual crystal structure of ice XIX having one of these space groups, although sufficient refinement agreements were obtained for the 18 candidates from our neutron diffraction data. A structural model of each candidate was constructed using a partially ordered model adopted in an earlier study ${ }^{8} . P \overline{4}$ or $P c c 2$ structural models are the most plausible for the space group of ice XIX, based on the structure refinements. Considering the suggested space group of ice $\mathrm{XV}, P \overline{1}^{14}$ or $P m m n^{8}$, centrosymmetry of hydrogen configurations is the most significance difference in hydrogen configuration between ice XIX and ice XV. In particular, Pcc2 suggests a pyroelectric structure as well as ice XI and its polar direction is along the $c$ axis. Although further investigations, such as a single-crystal neutron diffraction experiment, are necessary to precisely determine the hydrogen configurations, centrosymmetry will be an intriguing point in structural studies of ice XV and XIX.

Past arguments for the second hydrogen-ordered phase of ice VI should be mentioned here $7,13,15-17$. The existence of such a phase $\left(\beta-\mathrm{XV}^{13}\right)$ was suggested by Gasser et al. in samples decompressed from above $1.45 \mathrm{GPa}$ to ambient pressure using various measurements ${ }^{13}$. For example, differential scanning calorimetry (DSC) experiments show an endotherm peak which cannot be assigned to the known phase transition between ice VI and XV. Rosu-Finsen et al. ${ }^{15}$ reported detailed DSC experiments conducted at different heating rates and using different quenching/annealing procedures. Their DSC results clarified that the peak also appeared for quenched samples decompressed from 1.0 GPa, where ice XIX does not appear $^{13,15,16}$, and its appearance/disappearance depends on the heating rate of the DSC experiment. Based on these results, they questioned the supposed existence of a second hydrogen-ordered phase ${ }^{13}$, and explained the observed DSC profiles by introducing the idea of the deep-glassy state of ice VI. Meanwhile, based on the high-pressure observations presented herein, we firmly confirmed the presence of second hvdrocen-ordered bhase of ice VI. ice XIX. as a crystalline phase distinct from ice XV. In our Loading [MathJax]/jax/output/CommonHTML/fonts/TeX/fontdata.js 
view on the past arguments, although the samples decompressed from the higher-pressure region should have undergone hydrogen ordering from ice $\mathrm{VI}$ to ice $\mathrm{XIX}$, further investigation is necessary to confirm whether the samples retain the crystal structure of ice XIX at ambient pressure. This is because the idea of the deep-glassy state seems reasonable for the DSC results of their decompressed samples, and additionally on comparing our dielectric loss data to that measured under ambient pressure ${ }^{13}$, revival of reorientation dynamics, which should be immobilised upon hydrogen ordering ${ }^{2}$, was evidenced by a reappearance of dielectric loss in the decompressed samples ${ }^{13}$ (Supplementary Fig. 1b). This reactivated reorientation might partially break the long-range hydrogen order of ice XIX to obtain more stable configurations under lower pressure. It is necessary to ensure consistency among all the observed data, based on both the newly found ice XIX and the concept of the deep-glassy state.

This study first demonstrates the existence of multiple hydrogen-ordered phases for a hydrogendisordered phase and clarifies the effectiveness of applying pressure to induce phase competition among the hydrogen-ordered phases. Based on previous theoretical studies ${ }^{8-11}$ and the currently known phase diagram of ice, the low-temperature region of the phase diagram (below approx. $150 \mathrm{~K}$ ) is a frontier region for exploring undiscovered ways of hydrogen-ordering in ice, which would greatly change the phase diagram of ice. It is additionally noteworthy that the unit cell size of ice XIX allows many possible hydrogen-ordered configurations (1964 symmetry-independent configurations), such that an exhaustive theoretical analysis for the all configurations is difficult. However, such a wide variety of hydrogenordered configurations and their stability evaluations might be a good benchmark for modern theoretical trials toward modelling biochemical and environmental processes with large water molecules, such as using topological graph invariant theory ${ }^{20}$, combining oriented graph theory and density functional calculations, which can evaluate the energy stability of a large number of water-molecule arrangements. To the best of our knowledge, this is the first report in a hydrogen-bonded material for which different hydrogen-ordered configurations are realised depending on the pressure, although electric field is a known effective parameter to control ferro- and antiferroelectric structures of organic compounds ${ }^{21,22}$. This newly discovered coupling between hydrogen bond and pressure will extensively develop a new research field of the pressure-controllability of hydrogen-ordered configurations. Furthermore, by combining highelectric field with high-pressure, this multi-extreme condition is expected to provide more various types of hydrogen ordering and physical properties (e.g. (anti-)ferroelectricity) to hydrogen-bonded materials. Very recent technical development of neutron diffraction experiment ${ }^{23}$ will encourage such novel exploration in $P-T-E$ phase diagram focusing on the multiplicity of hydrogen-ordered phases.

\section{Methods}

\section{Dielectric measurements}

We conducted in-situ dielectric measurements under high pressure using a newly developed cell assembly. One of the most notable features of our development is that along with measuring the 
fluorescence method. This feature allows us to closely investigate the phase structure of ice in terms of its hydrogen ordering. The cell assembly is based on a piston-cylinder-type high-pressure apparatus (see details of the cell assembly in Supplementary information). In the dielectric experiments involving ice VI, $\mathrm{HCl}\left(99.9 \%\right.$, Wako) was introduced as a dopant (concentration: $\left.10^{-2} \mathrm{M}\right)$ to accelerate the hydrogen ordering of ice $\mathrm{VI}^{12}$, and dielectric experiments were conducted on $\mathrm{DCl}$-doped $\mathrm{D}_{2} \mathrm{O}$ ice $\mathrm{VI}(\mathrm{DCl}$ concentration: $10^{-2} \mathrm{M}$ ) following the same experiment procedure as that for $\mathrm{HCl}$-doped ice $\mathrm{VI}$ (Fig. 1). Pressure dependence of the phase transitions was similar to that of the $\mathrm{HCl}$-doped $\mathrm{H}_{2} \mathrm{O}$ sample, although the stable pressure region of the new hydrogen-ordered phase seemed to expand slightly to a lowerpressure region (between 1.3 and 1.5 GPa, see Supplementary Fig. 2).

\section{Neutron Diffraction Measurements}

The neutron diffraction measurements were conducted at PLANET beamline 11 at the Materials and Life Science Experimental Facility of J-PARC, Ibaraki, Japan ${ }^{24}$. DCl-doped $\mathrm{D}_{2} \mathrm{O}$ sample was used as a starting material ( $\mathrm{DCl}$ concentration: $10^{-2} \mathrm{M}$ ), and ice $\mathrm{VI}$ was prepared through solid-solid phase transitions, ice $\mathrm{III} \rightarrow \mathrm{V} \rightarrow \mathrm{VI}$, to obtain a fine powder sample (Fig. 1). Pressure and temperature were controlled by using a Mito-system ${ }^{25}$, and the pressure was estimated from the lattice parameter of $\mathrm{Pb}$, which was added to the sample as a pressure marker ${ }^{26}$.

\section{Structure Analysis Of Ice Xix}

Initial candidates of ice XIX were determined based on the group-subgroup relationship between ice VI and XIX, using the SUBGROUPS program opened on the Bilbao crystallographic server ${ }^{27-29}$. The structural models for the 18 space group candidates were constructed based on the partially hydrogenordered model adopted in a previous study ${ }^{8}$. Hydrogen occupancies and atomic coordinates were the fitting parameters obeying the ice rule in this model (details of the 18 structural models are given in Supplementary information). Structure refinements were conducted for the neutron diffraction patterns corrected at $1.6 \mathrm{GPa}$ and $80 \mathrm{~K}$ using all the structure models employing the Rietveld method (program: GSAS with EXPGUI ${ }^{30,31}$ ). We used the initial structural parameters with the ice VI structural model, based on the neutron diffraction pattern obtained at $1.6 \mathrm{GPa}$ and $80 \mathrm{~K}$. It was noted that site occupancies of hydrogen atoms were initially considered fitting parameters in the refinements with fixed atomic positions of hydrogen. $\chi^{2}$ values of the structure models are plotted in Supplementary Fig. 3, where numerical values are shown only for five candidates with $\chi^{2}<9$. For each structural model, Rietveld refinements

were performed several times to confirm their reproducibility. The space group $P \overline{4}$ was deemed the most plausible candidate in this step.

Supplementary Fig. 4 shows fitted lines using the five possible structural models (red-coloured) for the (black lines). It should be mentioned that an 
observed Bragg peak, marked by a black tick at $1.82 \AA$, shows broadening compared to the simulated ones. This peak is derived from the new hydrogen-ordered phase. Generally, peak broadening arises from two factors, insufficient crystallite size and/or microstrain in the crystal, consistent with the partially hydrogen-ordered state of ice XIX. Structure refinements, including atomic positions of hydrogen, were conducted for the five candidates (Supplementary Fig. 5); the fitted results obtained employing only hydrogen occupancies as fitting parameters (Supplementary Fig. 4) were subsequently used for these refinements. $\chi^{2}$ values of $\bar{P}$ and $P c C 2$ were comparable considering the dispersion of their refinement results, and those structural models were considered the most plausible for ice XIX. Atomic fractional coordinates for ice XIX using the two models are shown in Supplementary Tables 1 and 2, where lattice parameters have also been refined.

\section{Declarations}

\section{Acknowledgements}

We are grateful to the technical staff of the University of Tokyo (Graduate School of Science), Mr. S. Otsuka and Mr. T. Shimozawa, for their support in the experiments. Neutron diffraction experiments were performed using the J-PARC user program (proposal number 2019A0310). This research was supported by JSPS KAKENHI (Grant numbers: 19H00648, 18J13298, 18H05224, 18H01936, 15H05829).

\section{Author contributions}

R.Y. conceived and designed the experiments. R.Y., J.G., and Y.U. developed the high-pressure cell for dielectric measurements. R.Y., J.G., and H.I conducted the dielectric experiments. R.Y., K.K., S.M., and T.H. conducted the neutron diffraction experiments. R.Y. and K.K. analysed the neutron diffraction data. R.Y. wrote the manuscript with contributions from K.K., T.H., and H.K. All the authors have discussed the data interpretation.

\section{Competing interests}

The authors declare no competing interests.

\section{References}

1. Millot, M. et al. Nanosecond X-ray diffraction of shock-compressed superionic water ice. Nature 569, 251-255 (2019).

2. Whalley, E., Davidson, D. W. \& Heath, J. B. R. Dielectric properties of ice VII. Ice VIII: A new phase of ice. J. Chem. Phys. 45, 3976-3982 (1966).

3. Whalley, E. Dielectric Properties of Ice VII. Ice VIII: A New Phase of Ice. J. Chem. Phys. 45, 3976 (1966). 
4. Salzmann, C. G., Radaelli, P. G., Slater, B. \& Finney, J. L. The polymorphism of ice: Five unresolved questions. Phys. Chem. Chem. Phys. 13, 18468-18480 (2011).

5. Jackson, S. M., Nield, V. M., Whitworth, R. W., Oguro, M. \& Wilson, C. C. Single-crystal neutron diffraction studies of the structure of ice XI. J. Phys. Chem. B 101, 6142-6145 (1997).

6. W.F., K., Finney, J. L., Vettier, C. \& Bliss, D. V. Structure and hydrogen ordering in ices VI, VII, and VIII by neutron powder diffraction. J. Chem. Phys. 81, 3612 (1984).

7. Salzmann, C. G. Advances in the experimental exploration of water's phase diagram. J. Chem. Phys. 150, 60901 (2019).

8. Komatsu, K. et al. Partially ordered state of ice XV. Sci. Rep. 6, 28920 (2016).

9. Del Ben, M., Vandevondele, J. \& Slater, B. Periodic MP2, RPA, and boundary condition assessment of hydrogen ordering in ice XV. J. Phys. Chem. Lett. 5, 4122-4128 (2014).

10. Knight, C. \& Singer, S. J. Hydrogen bond ordering in ice v and the transition to ice XIII. J. Chem. Phys. 129,164513 (2008).

11. Tribello, G. A., Slater, B. \& Salzmann, C. G. A blind structure prediction of ice XIV. J. Am. Chem. Soc. 128, 12594-12595 (2006).

12. Salzmann, C. G., Radaelli, P. G., Hallbrucker, A., Mayer, E. \& Finney, J. L. The preparation and structures of hydrogen ordered phases of ice. Science 311, 1758-1761 (2006).

13. Gasser, T. M. et al. Experiments indicating a second hydrogen ordered phase of ice VI. Chem. Sci. 9, 4224-4234 (2018).

14. Salzmann, C. G., Radaelli, P. G., Mayer, E. \& Finney, J. L. Ice XV: A new thermodynamically stable phase of ice. Phys. Rev. Lett. 103, 105701 (2009).

15. Rosu-Finsen, A. \& Salzmann, C. G. Origin of the low-temperature endotherm of acid-doped ice VI: New hydrogen-ordered phase of ice or deep glassy states? Chem. Sci. 10, 515-523 (2019).

16. Thoeny, A. V., Gasser, T. M. \& Loerting, T. Distinguishing ice $\beta-X V$ from deep glassy ice VI: Raman spectroscopy. Phys. Chem. Chem. Phys. 21, 15452-15462 (2019).

17. Rosu-Finsen, A., Amon, A., Armstrong, J., Fernandez-Alonso, F. \& Salzmann, C. G. Deep-glassy ice VI revealed with a combination of neutron spectroscopy and diffraction. J. Phys. Chem. Lett. 11, 11061111 (2020).

18. Salzmann, C. G. et al. Detailed crystallographic analysis of the ice VI to ice XV hydrogen ordering phase transition. J. Chem. Phys. 145, 204501 (2016).

19. Kawada, S. Dielectric Dispersion and Phase Transition of KOH Doped Ice. J. Phys. Soc. Japan 32, 1442 (1972).

20. Mcdonald, S., Ojamae, L. \& Singer, S. J. Graph theoretical generation and analysis of hydrogenbonded structures with applications to the neutral and protonated Water cube and dodecahedral clusters. J. Phys. Chem. A 5639, 2824-2832 (1998).

21. Horiuchi, S. \& Ishibashi, S. Hydrogen-bonded small-molecular crystals yielding strong ferroelectric 
22. Horiuchi, S., Kumai, R. \& Ishibashi, S. Strong polarization switching with low-energy loss in hydrogenbonded organic antiferroelectrics. Chem. Sci. 9, 425-432 (2018).

23. Yamane, R. et al. Search for a ferroelectrically ordered form of ice VII by neutron diffraction under high pressure and high electric field. Phys. Rev. B 99, 174201 (2019).

24. Hattori, T. et al. Design and performance of high-pressure PLANET beamline at pulsed neutron source at J-PARC. Nucl. Inst. Methods Phys. Res. A 780, 55-67 (2015).

25. Komatsu, K. et al. Development of a new $\mathrm{P}-\mathrm{T}$ controlling system for neutron-scattering experiments. High Press. Res. 33, 208-213 (2013).

26. Strassle, T., Klotz, S., Kunc, K., Pomjakushin, V. \& White, J. S. Equation of state of lead from highpressure neutron diffraction up to $8.9 \mathrm{GPa}$ and its implication for the $\mathrm{NaCl}$ pressure scale. Phys. Rev. B 90, 14101 (2014).

27. Aroyo, M. I., Kirov, A., Capillas, C., Perez-Mato, J. M. \& Wondratschek, H. Bilbao Crystallographic Server. II. Representations of crystallographic point groups and space groups. Acta Crystallogr. Sect. A Found. Crystallogr. 62, 115-128 (2006).

28. Aroyo, M. I. et al. Bilbao Crystallographic Server: I. Databases and crystallographic computing programs. Zeitschrift fur Krist. 221, 15-27 (2006).

29. Aroyo, M. I. et al. Crystallography online: Bilbao crystallographic server. Bulg. Chem. Commun. 43, 183-197 (2011).

30. Toby, B. H. EXPGUI, a graphical user interface for GSAS. J. Appl. Crystallogr. 34, 210-213 (2001).

31. Larson, A. C. \& Von Dreele, R. B. GSAS: General Structure Analysis System. (2004).

\section{Figures}

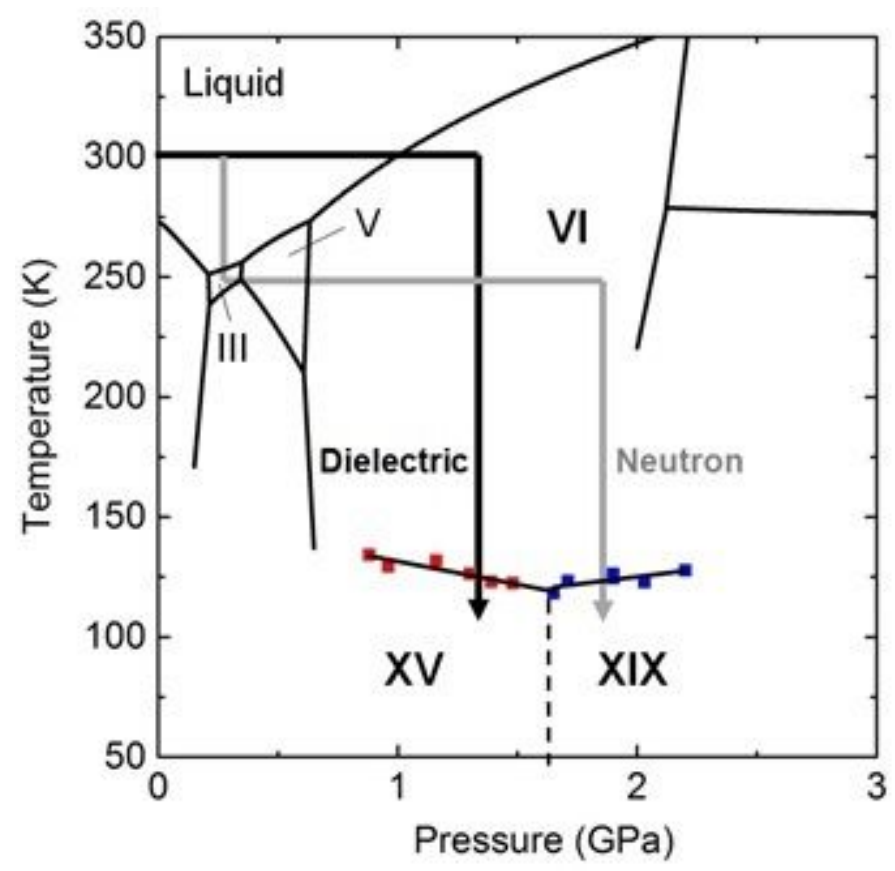


Representative experimental paths of dielectric and neutron diffraction experiments described in the phase diagram of ice obtained herein. Dielectric experiments of ice $\mathrm{VI}$ and its hydrogen-ordered phases were conducted at $0.88-2.2 \mathrm{GPa}$. $\mathrm{HCl}(99.9 \%$, Wako) was introduced as a dopant (concentration: 10-2 M) to accelerate the hydrogen ordering of ice VI12. The measured temperature was in the range $100-150 \mathrm{~K}$ and changed at a rate of $2 \mathrm{~K} / \mathrm{h}$. Neutron diffraction experiments of DCl-doped D2O (concentration: 10-2 $\mathrm{M}$ ) were conducted using a more complicated path to ensure that the sample was a fine powder through solid-solid phase transitions, i.e., ice III $\rightarrow$ ice $V \rightarrow$ ice VI. Sample diffraction was collected at 1.6 and 2.2 $\mathrm{GPa}$, and the temperature range was $80-150 \mathrm{~K}$. Temperature was changed at a rate of $6 \mathrm{~K} / \mathrm{h}$. Diffraction patterns were collected using new samples in each run at different pressures to confirm reproducibility. Phase boundaries among ice $V I$, ice $X V$, and ice $X I X$ are described by black solid lines, based on dielectric experiments (red and blue squares correspond to phase transition temperatures from ice $V I$ to ice $X V$ and $X I X$, respectively). The dotted line shows the provisional phase boundary between ice XV and ice XIX (see main text).
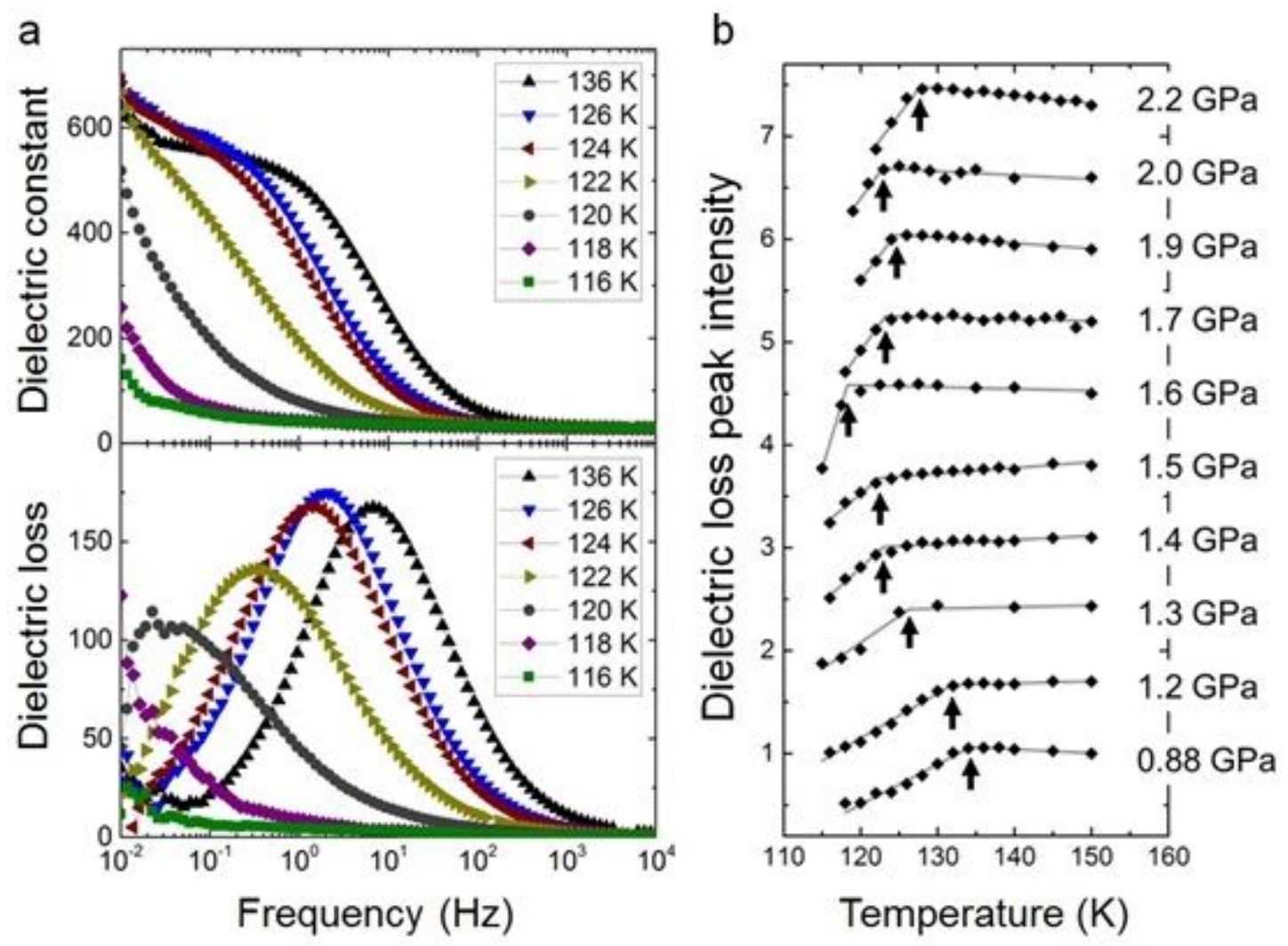

\section{Figure 2}

Temperature dependence of dielectric properties of $\mathrm{HCl}$-doped ice $\mathrm{VI}$ and its hydrogen-ordered phases. a, Dielectric constant and dielectric loss of $\mathrm{HCl}$-doped ice $\mathrm{VI}$ and its hydrogen-ordered phase (ice XIX) obtained at $1.9 \mathrm{GPa}$ upon cooling. The measured frequency was from $3 \mathrm{mHz}$ to $2 \mathrm{MHz}$. b, Temperature dependence of dielectric loss peak intensity of $\mathrm{HCl}$-doped ice $\mathrm{VI}$ and its hydrogen-ordered phases obtained in the pressure range 0.88-2.2 GPa upon cooling (black diamonds). Each peak intensity of dielectric loss was estimated using a model fitting the corresponding dielectric loss spectrum based on the Rehve dielentric-rolaxation enwation (nolvdisnersion type). Under each pressure, peak intensities were Loading [MathJax]/jax/output/CommonHTML/fonts/TeX/fontdata.js 
normalised by that obtained at the highest temperature. Each plot was shifted by 0.7 with increasing pressure for clarity. The grey lines were separately fitted for the data of loss peak intensity originating from ice $\mathrm{VI}$ and its hydrogen-ordered phases at each pressure. The phase-transition temperature was defined by an intersection of the two grey lines. The black arrows indicate the phase transition temperature at each pressure.
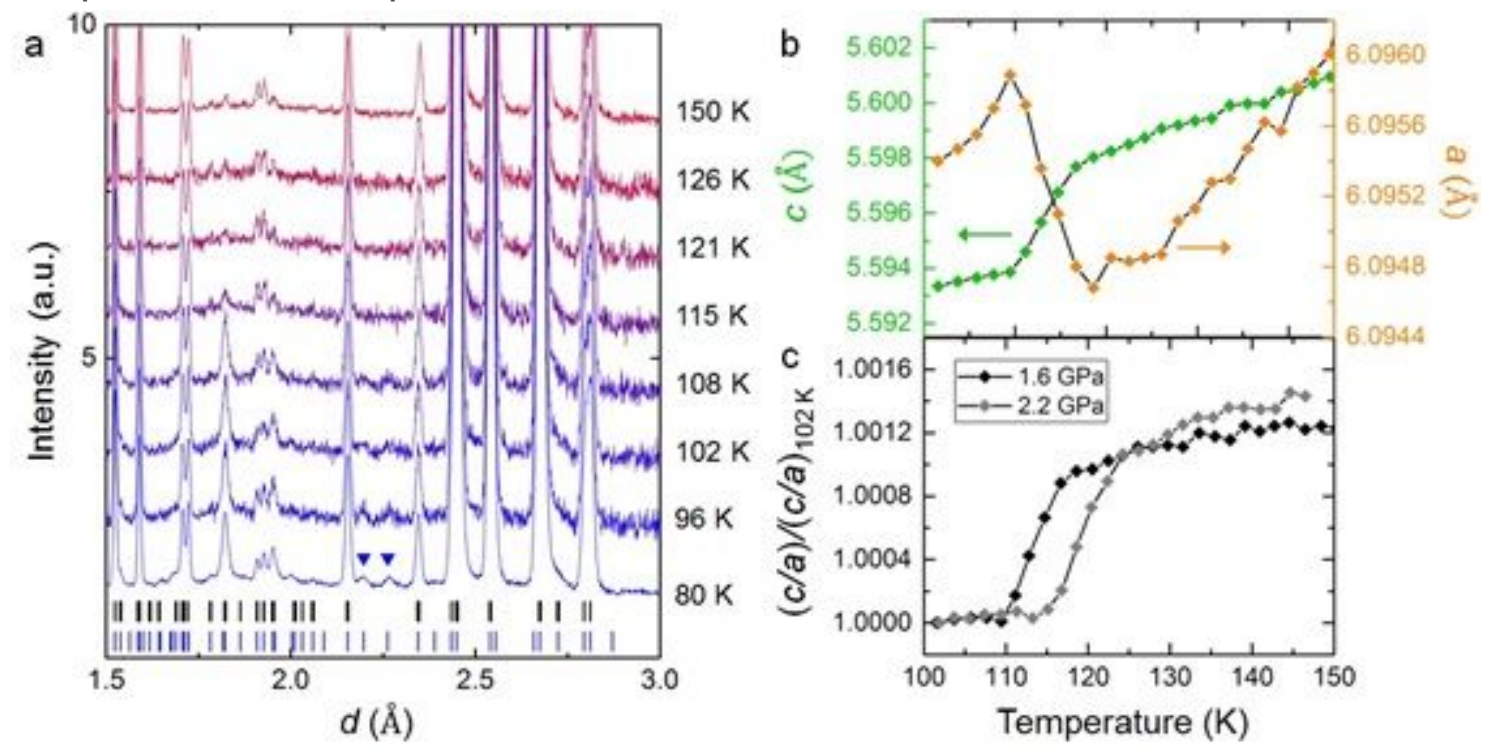

Figure 3

Temperature dependence of neutron diffraction patterns and lattice parameters of DCl-doped D2O ice VI and ice XIX. a, Neutron diffraction patterns of ice VI and XIX obtained at 1.6 GPa in the cooling run. Only an expanded area showing new peaks of ice XIX is displayed. The blue and black ticks represent all the peak positions expected from the unit cells of ice XIX and ice XV, respectively. Blue triangles indicate new peaks at $2.20 \AA$ and $2.26 \AA$, which do not appear from the unit cell of ice XV. b. Temperature dependence of lattice parameters, a and c, of ice VI or ice XIX obtained at 1.6 GPa. The values were calculated based on the ice VI structure model even for ice XIX because of a common oxygen framework between ice $\mathrm{VI}$ and XIX. c. Temperature dependence of c/a at two different pressures, 1.6 and $2.2 \mathrm{GPa}$, indicated by black and grey. Phase transition from ice VI to ice XIX started at around $117 \mathrm{~K}$ and $124 \mathrm{~K}$ in the respective cooling runs. The c/a values are normalised by that at $102 \mathrm{~K}$. Diffraction patterns were collected using new samples in each run under different pressures to confirm reproducibility.

\section{Supplementary Files}

This is a list of supplementary files associated with this preprint. Click to download.

- Supplementarymaterial.docx 